\title{
Discrimination and classification of rising and nonrising pitch patterns by the European starling (Sturnus vulgaris)
}

\author{
RICHARD F. BRAATEN, STEWART H. HULSE, and SUZANNE C. PAGE \\ Johns Hopkins University, Baltimore, Maryland
}

\begin{abstract}
In a series of four experiments, starlings learned to classify pitch patterns according to pattern structure. In Experiments 1 and 2, the starlings learned to discriminate a series of pairs of fourtone patterns in which one of the patterns rose in pitch and the other pattern did not. The starlings in these initial two experiments did not attend to pattern structure at all. Rather, they discriminated patterns according to the relative pitch of the initial pattern tones. In Experiment 3 , the same starlings discriminated the same patterns from Experiments 1 and 2 in a new context that tempered the salience of individual pattern tones. The starlings initially failed to discriminate in the new context, but eventually learned to do so. In Experiment 4, the starlings classified novel pitch patterns according to their pattern structure. These experiments identify a hierarchy of relative pitch strategies, as well as a sophisticated conceptual capacity for the perception and classification of pitch patterns by starlings.
\end{abstract}

In this report, we use new methods to show that a songbird, the European starling, can perceive pitch structure in tonal patterns. ${ }^{1}$

The importance of pattern structure in human auditory perception is widely accepted (Bregman \& Campbell, 1971; Divenyi \& Hirsh, 1978; Espinoza-Varas \& Watson, 1989; Garner, 1974; Garner \& Gottwald, 1968; Royer \& Garner, 1966, 1970). Generally, when listening to auditory patterns, humans are predisposed to perceive structure inherent in the relationships among pattern elements. Although the resolution of pattern elements can be improved under certain conditions (Espinoza-Varas \& Watson, 1986; Watson, Kelly, \& Wroton, 1976; Watson, Wroton, Kelly, \& Benbasset, 1975), the perception of pattern structure tends to dominate the perception of pattern elements in human auditory perception.

Music provides many examples of the perceptual primacy of pattern structure. In music, pattern structure is routinely transposed. Transposition maintains pattern structure by keeping the relationships among pattern elements intact. The stimulus characteristics of the individual elements themselves, however, are completely changed. Familiar transpositions in music include transpositions of melody, rhythm, and timbre. Despite these transpositions,

The research reported in this paper was supported by National Science Foundation Grants BNS 8606307 and BNS 8911046 to Stewart Hulse, and by a National Science Foundation Graduate Fellowship to Suzanne Page. We thank Curtis Kline for comments, and Christian Grue of the Patuxent Wildlife Preserve, Laurel, MD, for supplying the starlings. Correspondence concerning the paper should be addressed to Richard F. Braaten or Stewart H. Hulse, Department of Psychology, Johns Hopkins University, Baltimore, MD 21218. the perceptual identity of musical structure is maintained. The familiar tune is still familiar despite a shift in key. Rhythm is perceived to be constant, whether it is played at a faster or at a slower tempo. An oboe retains its characteristic timbre regardless of the pitch it produces.

In this report, we ask whether or not other animalsspecifically, European starlings-can perceive the pitch structure inherent in a simple melody. The human perception of melody is established by lawful variations in pitch, which are based on constant note-to-note frequency ratios. Melodies are perceptually invariant across any pitch transformation that maintains identical note-to-note pitch relationships, such as changes in key or octave shifts in tone height (Attneave \& Olson, 1971; Deutsch, 1982; Dowling \& Harwood, 1986). The fact that this perceptual invariance of melody has also been found in infants (Trehub, 1987) suggests that relational pitch perception may be an innate mode of human auditory perception.

Quite recently, research has addressed the question of whether nonhuman animals perceive tonal patterns on the basis of pitch relationships, as humans do in the perception of music (for reviews, see Hulse \& Page, 1988; Hulse, Page, \& Braaten, 1990). The results of this research have been equivocal. Songbirds have been shown to perceive pitch relations-in particular, rising as opposed to falling pitch patterns-but only as a secondary strategy (Hulse \& Cynx, 1985, 1986; Hulse, Cynx, \& Humpal, 1984; Page, Hulse, \& Cynx, 1989). Songbirds in these studies have preferentially discriminated tonal patterns according to the absolute frequency of the individual element tones in the patterns. Similarly, rats and cebus monkeys have been found to discriminate among tunes by attending to individual tones rather than pattern structure (D'Amato \& Salmon, 1982, 1984). 
It is somewhat surprising that songbirds have not proven more adept at perceiving structure in pitch patterns, given that songbirds communicate through complex pitch patterns. For example, starlings sing several whistled songs, which differ in contour and spectral structure (AdretHausberger, 1988). It would be surprising if the starlings were insensitive to the contour changes in their own songs. Recent studies have shown that, to some extent, songbirds do perceive pitch structure in conspecific song. Field sparrows have responded normally to playbacks of a test song that was shifted in overall frequency, as long as the frequency shifts left the song within the range of normal frequency for field sparrow song (Nelson, 1988). Blackcapped chickadees have displayed decreased responding to fee-bee songs with abnormal pitch relationships between the fee and bee elements (Weisman \& Ratcliffe, 1989). However, a strong effect of the absolute pitch of songs was also found in these studies. In the case of field sparrows, large frequency shifts resulted in significantly weaker responding. Similarly, chickadees showed decreased responding to fee-bee songs in which the frequencies of the fee and bee elements were changed, but the pitch relationship was maintained.

Most of the studies on tonal pattern perception have involved stimulus sets that allow the animal to discriminate among tonal patterns on the basis of either pattern elements or pattern structure. For example, Hulse et al. (1984) used four-tone rising and falling patterns, in which the rising patterns began on relatively low frequencies, and the falling patterns began on relatively high frequencies. Similarly, the tunes employed by D'Amato \& Salmon $(1982,1984)$ contained many frequencies that were unique to one of the melodies. In a recent study, Page et al. (1989) designed a stimulus set that required discrimination on the basis of pattern structure. In this stimulus set, the frequency of pattern elements was not correlated with tonal pattern structure. When faced with this stimulus set, starlings were unable to discriminate rising from falling pitch patterns even after many thousands of training trials. However, these same starlings were later trained to discriminate between rising and falling patterns in which pattern structure and the frequency of pattern elements were correlated, and were then presented with the uncorrelated stimulus set as unreinforced probe patterns. The starlings categorized the probe patterns according to both pattern structure and the absolute frequency of pattern elements. The results from the study by Page et al. suggest that unless distinctive absolute features are available in training, relational perception may not emerge. This suggests a preference hierarchy of pitch processing strategies, or, perhaps, a necessary learning sequence-from absolute features to general relations.

To date, the studies of perception of tonal patterns by songbirds have used procedures modeled after those used to study natural visual concepts in pigeons (Cerella, 1979; Herrnstein \& Loveland, 1964; Herrnstein, Loveland, \& Cable, 1976). The animal is presented with a pool of exemplars of the concept from the outset of training, and the pool is sampled on each individual trial. Thus, for example, Hulse et al. (1984) used 8 exemplars, and Page et al. (1989) used 64. However, the starlings were unable to learn a tonal pattern discrimination with this method when pattern structure and the frequency of pattern elements were uncorrelated. This led us to search for other methods to train a discrimination of tonal patterns.

An alternative method of discrimination training is to present individual discrimination problems successively, until each problem is mastered to a criterion. Animals trained in this fashion have been shown to develop a learning set (Harlow, 1949). The learning set is reflected by increasingly faster discrimination with each additional problem. Although the learning set method has traditionally been used to measure learning efficiency in nonclassification tasks (e.g., by Harlow, 1949; Herman \& Arbeit, 1973; Slotnick \& Katz, 1974), it could also be used to teach animals to classify stimulus patterns, in which each of a series of discrimination problems contains one member from each of two stimulus sets. Through exposure to a number of discrimination problems of this form, animals could presumably learn to discriminate on the basis of a classification rule that differentiates the members of the sets.

Our first experiment was based on a modified learning set method. Starlings were trained on a relatively small number of successive discrimination problems. Each discrimination problem contained two pitch patterns. One was structured according to a simple rule-four tones increased monotonically and by equal intervals on a log scale of frequency. The other pattern also contained four tones, but no consistent rule related successive pitches-the pitch contour (the "ups"' and "downs" in pitch) was haphazard. Furthermore, this haphazard pattern changed in contour from problem to problem. In effect, the starlings learned to distinguish and classify patterns with consistent as compared with inconsistent pitch contours. Subsequent experiments explored conditions that eventually led to relational discrimination of pitch patterns.

\section{EXPERIMENT 1 Initial Discrimination Training}

\section{Method}

Subjects. Two male (B39 and B44) and two female (B35 and W47) starlings (Sturnus vulgaris) served as subjects. All four starlings were caught in the wild and experimentally naive. The birds were obtained from the Federal Wild Life Preserve in Laurel, MD. They were maintained at $85 \%-90 \%$ of ad-lib weight on a diet of Purina Meat Builder, chopped dog chow, carrots, and hard-boiled eggs (including shells). Grit was available in the home cages, water was available at all times, and a water-vitamin solution was provided on alternate days in the home cage.

The birds were housed in an aviary containing 30-60 starlings. Fluorescent lighting in the aviary was controlled by a photocell and, later, by a Paragon EC72ST Sun Tracker. Both devices maintained the light:dark cycle consistent with that in Baltimore during the course of the experiment. Midway through the experiment, the birds were moved to a new aviary/laboratory. There were no major changes in housing conditions. 
Apparatus. The experimental apparatus consisted of a standard operant response panel mounted inside an Industrial Acoustics Company (IAC) acoustic chamber, Model AC-3, with internal measurements of $60 \times 80 \times 60 \mathrm{~cm}$. A three-sided weld-wire test cage measuring $28 \times 30 \times 20 \mathrm{~cm}$ was attached to the response panel, which formed the fourth side of the birds' test quarters. The response panel consisted of a row of three translucent response keys, $2 \mathrm{~cm}$ in diameter, and spaced $6 \mathrm{~cm}$ apart (center to center). Only the center and right response keys were used in this experiment. Pecks on the keys were detected by a microswitch. A food hopper delivered reward to a $6.0 \times 4.5-\mathrm{cm}$ opening located $4.5 \mathrm{~cm}$ below the center key. Indirect illumination (houselight) was provided by a translucent light source mounted on the side of the acoustic chamber. A 14-cm Jensen coaxial speaker was mounted in the ceiling of the IAC chamber, and centered $22 \mathrm{~cm}$ above the test cage.

Acoustic stimulus generation, control of experimental contingencies, and data recording were controlled by an IBM Personal Computer AT.

Pure sinusoid tones were created digitally at a sampling rate of $20 \mathrm{kHz}$. A Data Translation D/A converter fed computer output to a Coulborn Model S84-04 rise/fall gate set at a linear rise/fall time of $6 \mathrm{msec}$. Output from the gate was fed to a Crown D-75 amplifier, and then to the chamber speaker.

Stimuli. The stimuli for the six discrimination problems are shown in Figure 1. The 12 patterns that comprised the six problems were constructed of frequencies dividing the range of $1175-2960 \mathrm{~Hz}$ into eight equal intervals on a log scale. This frequency range is similar to that used in earlier studies (e.g., Hulse et al., 1984; Page et al., 1989), and is within the range of greatest auditory sensitivity for the starling (Dooling, Okanoya, Downing, \& Hulse, 1986). Each problem consisted of one pattern that rose consistently in pitch contour (the rising pattern) and one that varied haphazardly in pitch contour (a nonrising pattern). The contour of the rising patterns was based on a simple rule: Each successive tone was always one interval higher in pitch than the preceding tone. The nonrising patterns were structured so that the rising rule was violated (the pattern descended or remained the same in pitch) at least once in each pattern. The two patterns in each problem were constructed from the same four tones; patterns were distinguished by the serial order of those tones. For Problems B and C, one tone was repeated in the nonrising patterns. The mean frequency was thus slightly higher for the rising pattern of Problem B (rising, $1581 \mathrm{~Hz}$; nonrising, $1536 \mathrm{~Hz}$ ) and for the nonrising pattern of Problem $\mathrm{C}$ (rising, $1775 \mathrm{~Hz}$; nonrising, $1871 \mathrm{~Hz}$ ). The mean frequency of rising and nonrising patterns was identical for the other four problems.

Two birds (B35 and B39) learned the problems in the order A, B, C, D, E, F; to assess order effects, the other 2 (B44 and W47) learned in the order $A, B, F, E, D, C$.

All patterns, both rising and nonrising, maintained a common temporal structure. They were composed of four 100 -msec sine tones separated by 100 -msec intertone intervals. Each pattern presentation was separated by an 800 -msec interpattern interval. The total stimulus presentation period lasted until the bird made a response, or for $4 \mathrm{sec}$, whichever came first. Thus, each pattern could be presented a maximum of three times in the 4-sec presentation period.

Procedure. Initially, the starlings were trained to eat from the raised food hopper. The birds were then shaped to peck the lit center key, followed by a peck to the lit right key. After shaping, discrimination training began. The starlings were trained to discriminate the rising from the nonrising patterns in a go/no-go task. Each daily 2 -h session began with illumination of the houselight. The first trial began $5 \mathrm{sec}$ later with illumination of the center key. A peck at the center key darkened the key, illuminated the right key, and initiated presentation of one of the stimulus patterns. The structure of the pitch pattern was selected randomly $(p=.5)$, with the constraint that neither rising nor nonrising patterns be presented on more than three consecutive trials. If the pattern was a go stimulus, and the bird pecked the illuminated right key during the 4-sec presentation period, the key darkened, the sound pattern ended, and the bird had $3 \mathrm{sec}$ of access to the lighted food hopper. Failure to respond during the presentation period resulted in a 5-sec time-out period, during which the houselight was extinguished. If the pattern was a no-go stimulus, a response during the 4-sec presentation period resulted in the 5 -sec time-out period with extinguished houselight. If the bird did not respond during presentation of a no-go stimulus, the center key was illuminated, beginning the next trial. In any case, a 5-sec intertrial interval with the houselight on separated the trials.

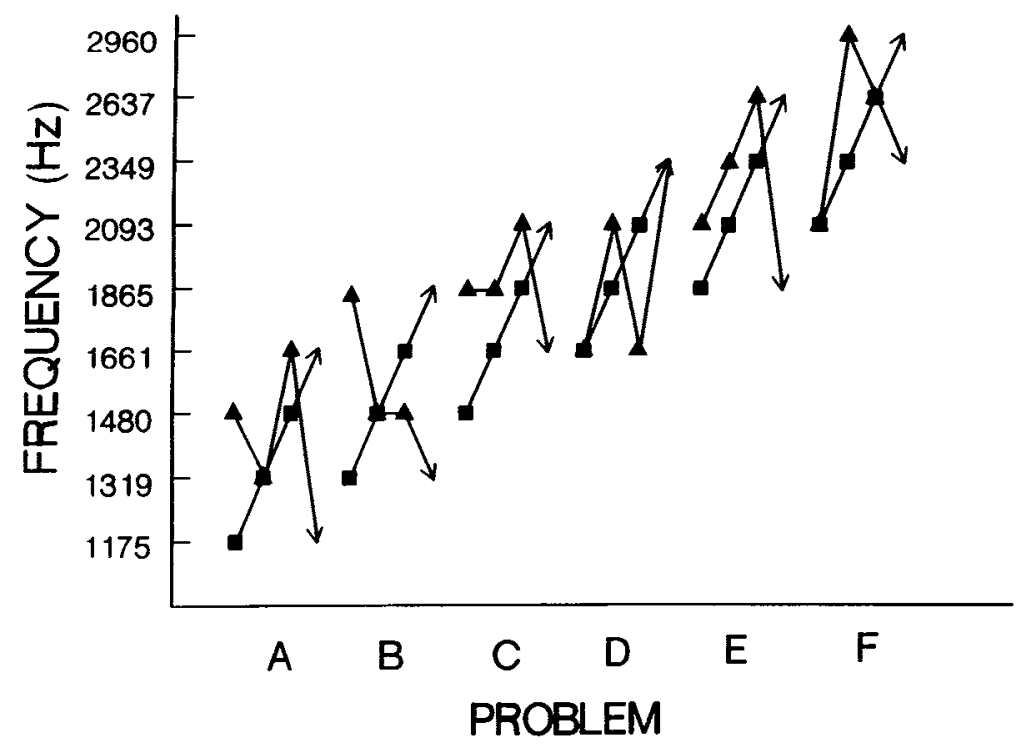

Figure 1. Rising and nonrising pitch pattern stimuli used for Experiment 1. Each discrimination problem contained a rising pattern and a monrising pattern. Rising pattern tones are represented by squares, and nonrising pattern tones are represented by triangles. 
Reinforcement contingencies were counterbalanced so that for 2 birds (B39 and W47), the rising patterns served as go stimuli, while for the other 2 birds (B35 and B44), rising patterns served as no-go stimuli. The birds were reinforced with food reward on $100 \%$ of correct go trials.

Stimulus problems were introduced in pairs of one rising and one nonrising pattern. Each problem was presented until the bird was discriminating at a criterion of $85 \%$ correct for two out of three consecutive sessions. A response was scored as correct if the bird pecked the lighted key during the 4-sec presentation period of a go stimulus, or withheld pecking for $4 \mathrm{sec}$ during presentation of a no-go stimulus. Otherwise, the response was scored as incorrect.

Once a problem had been learned to criterion, a new problem, consisting of one rising and one nonrising pattern, was presented. This sequence was continued until the birds had learned all six discrimination problems.

For 3 birds (B35, B39, and W47), each problem (except for the first one) was followed by one or two sessions in which all patterns that had been learned up to that time were presented within the same session. On any given trial in these intervening sessions, a peck on the center key randomly selected a pattern for presentation ( $p=1 /$ number of patterns). The 4 th bird (B44) did not receive this intervening session between problems; the six discrimination problems followed one after the other without interruption.

\section{Results and Discussion}

The starlings were able to discriminate rising from nonrising patterns. All four birds learned to discriminate to the criterion of $85 \%$ correct in all six of the problems for two out of three consecutive sessions.

If the starlings were learning to discriminate the patterns on the basis of the consistent tonal structure that distinguished them, positive transfer to new discrimination problems would be expected. Positive transfer should arise because the same structural relationships obtained between the two patterns for each of the six problems. Table 1 shows the number of trials required for each bird to reach criterion on each of the six problems. Although there was a small negative correlation between the order in which the problem was learned and trials to criterion (Pearson's $r=-.28)$, the correlation was not significant $[F(1,22)=$ $1.87, p>.05]$. Initial training thus provided no evidence for the perception of pattern structure.

Table 2 shows discrimination performance during the sessions intervening between new problems for the 3 birds that received them. These sessions contained all the problems that had been learned up to that time. Table 2

Table 1

Trials to Criterion for the Six Discrimination Problems of Experiment 1 as a Function of Problem Order

\begin{tabular}{ccrrrrrr}
\hline & & \multicolumn{2}{c}{ Bird } & & \multicolumn{2}{c}{ Bird } \\
\cline { 3 - 4 } \cline { 7 - 8 } Order & Problem & B35 & \multicolumn{1}{c}{ B39 } & Problem & B44 & W47 \\
\hline 1 & A & 9,303 & 11,243 & A & 13,259 & 9,004 \\
2 & B & 2,000 & 2,765 & B & 1,790 & 1,190 \\
3 & C & 1,000 & 1,000 & F & 14,699 & 3,557 \\
4 & D & 7,667 & 12,470 & E & 6,456 & 3,206 \\
5 & E & 5,373 & 4,500 & D & 8,889 & 3,046 \\
6 & F & 3,386 & 2,398 & C & 5,360 & 2,681 \\
\hline
\end{tabular}

Note-B35 and B39 received the problems in the order A, B, C, D, E, F. B44 and W47 received the problems in the order A, B, F, E, D, C.
Table 2

Percentages of Correct Responses on Intervening Sessions as a Function of Number of Problems Learned

\begin{tabular}{cccc}
\hline \multirow{2}{*}{$\begin{array}{c}\text { Number } \\
\text { of Problems }\end{array}$} & B35 & B39 & W47 \\
\hline \cline { 2 - 4 } & 96 & 90 & 94 \\
3 & 80 & 82 & 66 \\
4 & 52 & 53 & 62 \\
5 & 63 & 57 & 53 \\
6 & 53 & 54 & 55 \\
\hline
\end{tabular}

Table 3

Percentages of Correct Responses on the First Session of the Six Discrimination Problems of Experiment 1

\begin{tabular}{ccccc}
\hline & \multicolumn{4}{c}{ Bird } \\
\cline { 2 - 5 } Problem & B35 & B39 & B44 & W47 \\
\hline A & 47 & 50 & 49 & 49 \\
B & 74 & 83 & 73 & 94 \\
C & 92 & 93 & 50 & 55 \\
D & 49 & 48 & 54 & 54 \\
E & 52 & 49 & 51 & 48 \\
F & 51 & 52 & 51 & 49 \\
\hline
\end{tabular}

shows performance on the first such session following each problem. The birds maintained good discrimination on up to three problems, but once the fourth problem was added, discrimination declined almost to chance. This finding provides further evidence that the birds were not discriminating on the basis of the tonal structure that distinguished the two sets of patterns.

Although there was no improvement in learning across problems in general, the starlings found some problems much easier to learn than others. In some cases, there was almost perfect transfer from one problem to the next. Furthermore, performance on transfers depended on which of the two specific problem orders the starlings experienced. Table 3 shows the percentage of correct responses for each bird on the first session of each of the six problems. All birds showed clear evidence of discrimination on the very first session for Problem B. Two birds, B35 and B39, also showed good discrimination performance on the very first session of Problem $C$.

In each case in which a bird showed good discrimination performance on the very first session of a new problem, the pitch relationship between the initial tone of the two patterns in the new problem was the same as that in the problem that had just been learned. For example, the initial frequency in the nonrising pattern of Problem B was higher than the initial frequency of the rising pattern. This same pitch relationship held for the immediately preceding Problem A. Similarly, the nonrising pattern of Problem $\mathrm{C}$ had a higher initial frequency than did the rising pattern. Two of the birds, B35 and B39, received this problem following Problem $B$, and they showed good discrimination. The other 2 birds, B 44 and W47, received this problem following Problem D, which did not have the same initial frequency relationship, and they showed poor initial discrimination. 
Statistical analyses of the percentage of correct responses on the first session of each problem (cf. Table 3) support these conclusions. A mixed two-factor analysis of variance, with problem order as the between-groups factor and the five problems B-F as the within-subjects factor, showed that the interaction between the two problem orders and performance on the first session of the five problems was significant $[F(4,8)=12.26, p<.01]$. Problem A was omitted from the analysis because the birds had no prior experience with the experimental contingencies.

The interaction can be accounted for by the superior performance of B35 and B39 on Problem C. An analysis of simple effects on order for each discrimination problem showed that the specific sequence of the problems had a reliable effect for Problem $\mathrm{C}$, in which the pitch relationship of initial tones was maintained for B35 and B39, but not for B44 and $\mathrm{W} 47[F(1,2)=246.15, p<.01]$. The small difference in performance between the two groups on Problem D was also significant $[F(1,2)=121.00$, $p<.01]$. While the pitch relationship of the initial tones of Problem D was not the same as it was in the preceding problem, the difference in discrimination performance was very small. There was no difference between the groups for Problems B, E, or F, in which the pitch relationship of the initial tones from problem to problem was the same between both groups of birds $[F \mathrm{~s}(1,2)=.19$, .22 , and 1.80 , respectively, $p s>.05$ ].

To summarize, Experiment 1 showed that starlings could learn a discrimination between rising and nonrising pitch patterns, but there was no evidence of steady improvement in performance from problem to problem. The starlings did not learn the discrimination on the basis of the tonal pattern structure. Of course, the starlings only learned six problems, perhaps an insufficient number. Nevertheless, there was significant evidence for the transfer of a relational discrimination from problem to problem - not on the basis of overall pattern structure (i.e., rising as compared with nonrising) but rather on the basis of pitch relationships between the initial tones of the patterns. The starlings' solution to the problem was good, given the experimental contingencies, because it allowed them to respond immediately, rather than wait for the entire pattern to unfold over time. These results indicate that starlings do possess the capacity for relative pitch perception in this restricted form. This finding provided the rationale for the additional transfer problems of Experiment 2 .

\section{EXPERIMENT 2 \\ A Test for Relational Perception Based on Initial Tone Frequencies}

Although the starlings appeared to be discriminating the patterns of Experiment 1 according to the ordinal pitch relationships of the initial tones of the patterns, the data from that experiment were not conclusive. An alternative possibility was that the birds at first used ordinal pitch relationships to solve the discrimination problems, and then began using pitch relationships among the tones within the patterns when the original strategy no longer worked. This would have occurred with Problem D for starlings B35 and B39, and Problem F for starlings B44 and W47. In these problems, the initial tones of the rising and nonrising patterns were the same, so that there was no ordinal pitch information in the initial tones. Support for this idea came from the gradual improvement in discrimination following these problems, even when both patterns of the problem contained the same initial tone, as in Problem F for B35 and B39, and Problem D for B44 and W47.

To test among these alternative explanations, three new problems were constructed. It was hypothesized that if the pitch relationship between the initial tones was important, then transfer to problems in which this relationship was maintained would be better than transfer to problems in which the relationship was reversed. If the pitch relationship among tones within a pattern was important, then transfer to new problems would be good as long as that pitch relationship was maintained.

\section{Method}

Subjects and Apparatus. The subjects and apparatus were the same as in Experiment 1.

Stimuli. The three novel problems consisted of various transpositions of one of the initial problems, Problem C. Contour and interval relationships between the tones in the patterns remained the same; only the frequencies changed.

The three transfer problems, along with the baseline problem (Problem C), are shown in Figure 2. In Problem G, the rising pattern from Problem $C$ was transposed up two whole-tone intervals on the log scale of frequency, and the nonrising pattern was transposed down two intervals. This reversed the frequency relationship of the initial tones of the rising and nonrising patterns in Problem C; the rising pattern in Problem $G$ began on a higher frequency than did the nonrising pattern. Problems $\mathrm{H}$ and $\mathrm{I}$ retained the frequency relationship of the initial tones in Problem C. In Problem $\mathrm{H}$, both the rising and the nonrising baseline patterns were transposed up one interval in frequency, and in Problem I, both patterns were transposed up four intervals.

Procedure. Each bird was retrained on the baseline problem, Problem C (see Figure 2). Once performance on Problem C reached $85 \%$ correct for two consecutive sessions, the birds were transferred on the very next session to one of the three transfer problems. Reinforcement contingencies remained the same as in the initial six discrimination problems. The birds were presented with the transfer problem until the criterion of $85 \%$ correct for two consecutive sessions was reached. At this point, the birds were transferred back to Problem C. After recovering criterion performance on Problem C, the birds were transferred to the second transfer problem, remaining on that problem until criterion was reached. This procedure continued until the birds had transferred from Problem $C$ to all three of the transfer problems, and had learned them all to criterion.

Two birds (B39 and W47) received the problems in the order C, G, C, H, C, I, and the other two birds (B35 and B44) received the problems in the order $\mathrm{C}, \mathrm{H}, \mathrm{C}, \mathrm{G}, \mathrm{C}, \mathrm{I}$.

\section{Results and Discussion}

The starlings performed better on the two problems $(\mathrm{H}$ and I) in which the high-low frequency relationship be- 


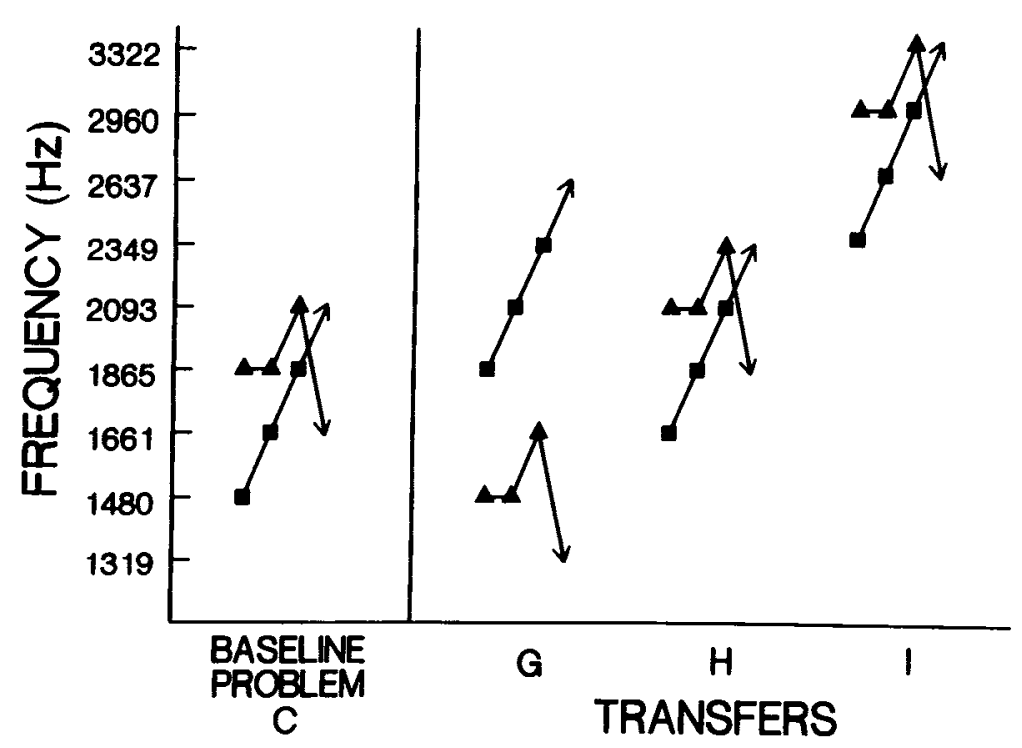

Figure 2. Rising and nonrising pitch pattern stimuli used for Experiment 2. Each of the three transfer problems $(G, H$, and $)$ was a transposition of a baseline problem, Problem C.

tween the initial tones of the patterns was maintained than they did on the problem $(G)$ in which the relationship was reversed. The percentage of correct responses for each of the birds on the first session of Problems G, H, and $I$ are shown in Table 4. All birds performed at chance on the first session of Problem G, in which the pitch relationship of the initial tones was reversed. However, all 4 starlings showed excellent transfer to the small oneinterval transposition of Problem $\mathrm{H}$. In addition, 2 of the birds, B35 and B44, were able to discriminate among the patterns even with the large, four-interval transposition of Problem I.

The first $\mathbf{5 0 0}$ trials of each of the three transfers were analyzed in 10 blocks of 50 trials. Pairwise comparisons showed that discrimination was better on transfers that maintained the initial-tone pitch relationship (Problems $\mathrm{H}$ and I) than on Problem $\mathrm{G}$, where this relationship was reversed. All 4 starlings performed significantly better over the first 500 trials of Problem $\mathrm{H}$, a one-tone transposition that maintained the initial-tone pitch relationship, than on Problem G, in which the initial-tone pitch relationship was reversed $[F \mathrm{~s}(1,9)=190.32,222.15,318.78$, and 45.80 for B35, B44, B39, and W47, respectively, all $p s<.01]$. Similarly, 3 of the birds performed better over the first 500 trials of the larger four-tone transposition of Problem I than they did on Problem G $[F(1,9)=26.41$,

Table 4

Percentages of Correct Responses on the First Session of the Three Transfer Problems of Experiment 2

\begin{tabular}{ccccc}
\hline & \multicolumn{4}{c}{ Bird } \\
\cline { 2 - 5 } Problem & B35 & B39 & B44 & W47 \\
\hline G & 50 & 51 & 48 & 46 \\
H & 94 & 96 & 97 & 90 \\
I & 77 & 57 & 72 & 52 \\
\hline
\end{tabular}

and 20.68 for B35 and B44, respectively, $p s<.01$; $F(1,9)=5.47$ for B39, $p<.05]$. Starling W47 did not perform better on Problem I than on Problem G $[F(1,9)=$ $.44, p>.05]$.

The results from these transfers support the conclusion that the discrimination was based on the pitch relationship between the initial tones of the patterns. The contour of the rising and nonrising patterns was exactly the same in each of the three problems, yet transfer performance differed. When the high-low pitch relationship of the initial tones was consistent from problem to problem, transfer was generally good. When the high-low pitch relationship was inconsistent, transfer was poor.

Transfer was better for the small transposition of Problem $\mathrm{H}$ than for the large transposition of Problem I. This indicates that the pitch relationship of the initial tones is not all that was involved in the discrimination. The size of the frequency displacement also had an effect. In fact, one of the frequencies used in Problem I was outside the range of the frequencies used in training. The poor transfer to this problem, then, may have been a result of the frequency-range constraint. Under the frequency-range constraint, songbirds lose a pitch pattern discrimination if frequencies are shifted outside the frequency range in which the discrimination has been learned (Cynx, Hulse, \& Polyzois, 1986; Hulse \& Cynx, 1985).

The results demonstrate that starlings are capable of relative pitch perception. However, the type of relative pitch perception employed by the starlings in this experiment is different from the type of relative pitch perception used by humans in the perception of melodies. The starlings appeared to be solving the discrimination according to the relative "highness" or "lowness" of the initial tone of one pattern as compared with the initial tone of the other. 
Although relative pitch perception is necessary for classification of pitch patterns, such classification also requires competence with temporal order. In order to classify different exemplars of a pitch pattern, the starlings must be able to judge the pitch relationships of successive tones within a pattern. The temporal order of these pitch relationships defines the pattern structure. Specifically, in the present experiments, the structure was defined as "rising, rising, rising." Any nonrising relationship between successive tones violated this structure. There is no evidence that the starlings learned anything about pitch pattern structure in Experiments 1 or 2.

\section{EXPERIMENT 3 \\ Perception of Pitch Patterns in a Context Eliminating Initial-Tone Cues}

The results from the previous two experiments showed no evidence of discrimination on the basis of pitch structure. Rather, the starlings attended to the initial tones of the patterns. In Experiment 3, the predictive value of individual tones was reduced to test whether or not starlings could be induced to attend to the overall structure of a pattern when their preferred single-tone strategy was no longer adequate.

The initial six discrimination problems contained numerous pitch cues other than contour, which could be used by the starlings to maximize reinforcement. Results thus far suggested special significance for one of these cues, the relative pitch of the initial tones. It was hypothesized that if this was true, then presenting all 12 of the patterns within a single session would pose a considerable challenge to the birds. Cues that had been reliable in the context of the sequential problems would no longer be useful when the problems were presented simultaneously. For example, in Problem C (see Figure 1), the initial frequency of the nonrising pattern $(1865 \mathrm{~Hz})$ was greater than the initial frequency of the rising pattern $(1480 \mathrm{~Hz})$. But when all 12 patterns were presented within a session, rising and nonrising patterns were initiated by both 1865 and $1480 \mathrm{~Hz}$, rendering this cue unreliable. Perhaps by diminishing the distinctiveness of these cues, perception of the tonal structure of the patterns would be induced in the starlings.

\section{Method}

Subjects and Apparatus. The subjects and apparatus were the same as in Experiments 1 and 2.

Stimuli. The stimuli were the 12 patterns shown in Figure 1.

Procedure. The birds received daily sessions consisting of all 12 of the initial stimulus patterns shown in Figure 1. One of these twelve patterns was randomly selected for presentation on each trial ( $p=.083$ for each pattern). Contingencies were the same as in Experiment 1.

\section{Results and Discussion}

Although all birds had learned to discriminate each of the problems to the criterion of $85 \%$ correct during Experiment 1 , they were at first unable to discriminate among the same pitch patterns when they were presented together within a single session. Mean latencies for each bird to go and no-go patterns on the first and last sessions of Experiment 3 are shown in Table 5. The number of sessions intervening between the first and last session ranged from 142 for B44 to 176 for B39. There was no difference in the latency to respond to go patterns as opposed to no-go patterns during the first session $[F(1,3)=$ $2.37, p>.05]$. The birds did, however, eventually learn to discriminate rising from nonrising patterns within this context. Latencies were significantly shorter for go as opposed to no-go patterns during the last session $[F(1,3)=$ $69.07, p<.01]$.

The birds found some patterns more difficult to classify than others. Table 6 shows the mean percentage of correct responses for each bird for each pattern, averaged over the last three sessions of Experiment 3. Table 6 reveals a response bias that was present throughout the first three experiments. The starlings were much more likely to commit errors on no-go trials than on go trials. When unable to discriminate among patterns, the birds tended to go for all patterns. This is probably due to the fact that food was only available on go trials. Thus, percent correct in Table 6 is much higher for go than for no-go patterns.

Although the more difficult patterns vary somewhat from bird to bird, there are some consistencies. The rising patterns of Problems $E$ and $F$ were relatively difficult for the 2 birds, B35 and B44, that were trained with no-go contingencies for rising patterns. The nonrising pattern

Table 5

Mean Latencies to Respond to Go and No-Go Patterns on the First and Last of the Simultaneous Problem Sessions of Experiment 3

\begin{tabular}{|c|c|c|c|c|}
\hline \multirow[b]{2}{*}{ Pattern Type } & \multicolumn{4}{|c|}{ Bird } \\
\hline & B35 & B39 & B44 & W47 \\
\hline \multicolumn{5}{|c|}{ First Session } \\
\hline Go & .74 & .36 & .87 & .78 \\
\hline No-Go & 1.14 & .39 & 1.02 & .77 \\
\hline \multicolumn{5}{|c|}{ Last Session } \\
\hline & .76 & .57 & 1.21 & 1.43 \\
\hline No-Go & 2.62 & 2.01 & 2.59 & 2.46 \\
\hline
\end{tabular}

Note-Latencies are represented in seconds.

Table 6

Mean Percentages of Correct Responses on the 12 Pitch Patterns of Experiment 3

\begin{tabular}{|c|c|c|c|c|c|c|c|c|c|c|c|c|}
\hline \multirow[b]{3}{*}{ Bird } & \multicolumn{12}{|c|}{ Patterns } \\
\hline & \multicolumn{6}{|c|}{ Rising } & \multicolumn{6}{|c|}{ Nonrising } \\
\hline & A & B & $\mathrm{C}$ & D & $\mathbf{E}$ & $\mathbf{F}$ & $\mathbf{A}$ & B & C & D & $\mathbf{E}$ & $\mathbf{F}$ \\
\hline \multicolumn{13}{|c|}{ Nonrising $=\mathrm{Go}$} \\
\hline B35 & 86 & 91 & 79 & 48 & 44 & 33 & 97 & 100 & 99 & 98 & 97 & 98 \\
\hline B44 & 66 & 81 & 55 & 60 & 46 & 53 & 92 & 93 & 82 & 86 & 85 & 86 \\
\hline \multicolumn{13}{|c|}{ Nonrising $=$ No-Go } \\
\hline B39 & 100 & 100 & 100 & 98 & 99 & 96 & 36 & 75 & 10 & 8 & 2 & 84 \\
\hline W47 & 90 & 97 & 96 & 86 & 80 & 82 & 45 & 73 & 50 & 50 & 21 & 71 \\
\hline
\end{tabular}

Note-Percentages represent averages over the last three sessions of Experiment 3. 
of Problem E was difficult for the 2 birds, B39 and W47, that were trained with go contingencies for rising patterns.

The reason for this differential discriminability of problems is not readily apparent. For example, the nonrising pattern of Problem $E$ and the rising pattern of Problem $F$ were identical except for the last tone. The birds found these patterns difficult to classify. This difficulty could have arisen because the patterns were similar in contour, or because the absolute frequencies in the patterns were similar. Thus the differential difficulty of the patterns does not clearly discriminate classification based on absolute features from that based on pitch pattern structure.

In summary, the birds learned to discriminate on all six problems to criterion when the problems were presented one at a time in Experiment 1, but they first performed at chance when all of the problems were presented within a session. This provides additional evidence that pitch contour did not play a part in the starlings' discrimination on the problems in the first two experiments. If pitch contour had been controlling responding, the starlings should have readily categorized rising and nonrising patterns on the basis of their common pattern structuresespecially given that they had already learned to do so on a problem-by-problem basis. Instead, the dearth of reliable cues from individual tones in this new context produced substantial confusion.

The birds did eventually gain proficiency in discriminating rising from nonrising problems in the context of Experiment 3. Two explanations are possible. When the pitch relationship of the initial tones was greatly diminished as a discriminative cue, the birds may have learned to use pitch structure to discriminate the patterns. Alternatively, the birds may have learned to use more sophisticated concatenations of absolute features. These possible explanations prompted Experiment 4.

\section{EXPERIMENT 4 Probes with Novel Patterns}

In order to test the possibility that the birds had learned to discriminate the pitch patterns by pattern structure once other cues had been reduced, novel probe patterns were presented within the simultaneous problem sessions. It was hypothesized that if the birds had learned to categorize the patterns according to tonal structure, then they would be able to categorize new problems with the same tonal structure. If, however, they had learned to sort the patterns according to isolated tones, or to combinations of tones specific to those patterns, then they would not be able to sort the novel patterns.

\section{Method}

Subjects and Apparatus. The subjects and apparatus were the same as in the previous experiments.

Stimuli. Twenty-four probe patterns were constructed of frequencies spaced at equal log intervals in the range of $523-5586 \mathrm{~Hz}$. They were within the starling's range of audibility (Dooling et al., 1986). Half of the probes (12) were constructed from frequencies within the $1175-2960-\mathrm{Hz}$ frequency range of the 12 baseline patterns; these are shown in Figure 3 . The other half contained frequencies outside-either above or below-that range; these are shown in Figure 4.

There were three types of probes both inside and outside the baseline frequency range. Eight of the probes were novel rising patterns. These probes, shown in the left panels of Figures 3 and 4 ,

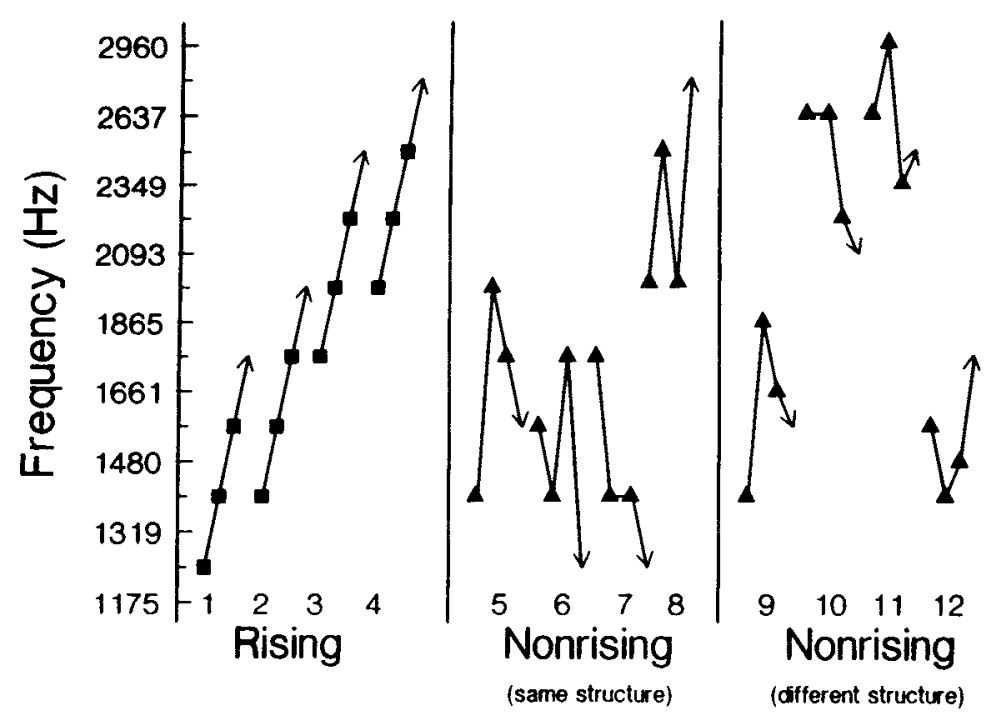

Figure 3. Rising and nonrising probe patterns used in Experiment 4. These 12 patterns were constructed from frequencies within the 1175-2960-Hz frequency range of the 12 baseline patterns. The left panel shows the 4 novel rising patterns. The center panel shows the 4 novel nonrising patterns that were exact transpositions of baseline patterns. The right panel shows the 4 novel nonrising patterns that were not exact transpositions of baseline patterns. 


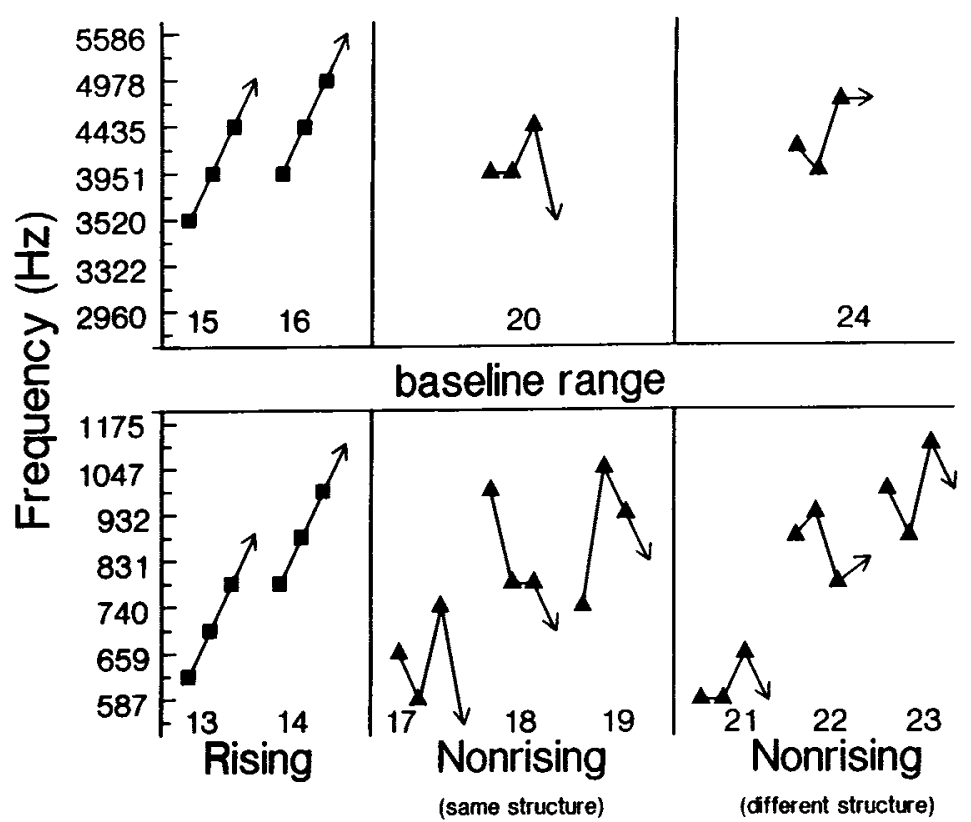

Figure 4. Rising and nonrising probe patterns used in Experiment 4. These 12 patterns were constructed from frequencies outside the $1175-2960-\mathrm{Hz}$ frequency range of the 12 baseline patterns. The left panel shows the 4 novel rising patterns. The center panel shows the 4 novel nonrising patterns that were exact transpositions of baseline patterns. The right panel shows the 4 novel nonrising patterns that were not exact transpositions of baseline patterns.

were exact transpositions of the 12 baseline patterns from Figure 1. They maintained the same contour and interval relationship between tones as did baseline rising patterns, but contained novel frequencies. Sixteen of the probes were novel nonrising patterns. Within these 16 patterns, there were two types. Eight of the patterns, shown in the center panels of Figures 3 and 4, were exact transpositions of the structure of the nonrising baseline patterns from Figure 1. Like the novel rising probes, they retained the same contour and interval relationship between tones; only the frequencies changed. The other 8 nonrising probes, shown in the right panels of Figures 3 and 4 , were not exact transpositions. That is, their structures varied in either interval or contour from the 6 nonrising baseline patterns.

All probe patterns retained the same temporal structure as the baseline patterns.

Procedure. Once the starlings had reached stable levels of performance on the simultaneous problems, they were exposed to the series of novel rising and nonrising probe patterns. During each daily probe session, the birds were presented with the 12 original baseline patterns from Figure 1, plus one unique probe pattern. Thus, on any given trial, the birds were exposed to 1 of 13 patterns $(p=.077)$. Correct responses on the 12 baseline patterns were reinforced with $p=.80$. Responses on the probe patterns were not reinforced. To reduce the amount of information available from reinforcement contingencies on probe trials, correct responses for no-go baseline patterns were also reinforced with $3 \mathrm{sec}$ of access to food. In the preceding experiments, these trials had not been reinforced. This step was taken, because otherwise, the contingencies for not responding on probe trials would have been exactly the same as the contingencies for not responding on no-go baseline trials, and this might have biased the birds to not respond on the probe trials.

Each starling was exposed to all 24 probe patterns. A single probe pattern was presented in consecutive sessions until three criteria were met within a single session. First, the birds were required to complete at least 150 trials in a single session. Second, the birds had to receive at least 15 probe trials within the session. Third, performance on the 12 baseline patterns had to be at least $60 \%$ correct. When these three criteria were met, a bird moved on to the next probe. The probes were presented in a different random order for each bird. Of the 152 total sessions completed by the four birds, 96 (63\%) met these criteria. Thus, most probes were presented for only one session.

\section{Loudness Transfer}

A loudness control was incorporated into this experiment. Because loudness had neither been equated exactly nor randomized in these experiments, it is possible that the birds were discriminating the patterns on the basis of the loudness of the component tones. Although similar experiments have not revealed an effect for loudness (Hulse \& Cynx, 1985; Hulse et al., 1984; Page et al., 1989), a loudness transfer test was conducted to control for this possibility.

The four starlings were presented with the 12 simultaneous problem sessions, until performance stabilized. At this point, amplitude of the component tones was measured by a Rion Model NA-20 loudness meter, with its microphone placed at the approximate position of a bird's head as it pecked the center key. Amplitude of the tones was measured to range from $62-71 \mathrm{~dB}(\mathrm{~A})$. On the following session, birds were transferred to the same 12 patterns in which the tones were roughly equated for amplitude in a range of $67-68 \mathrm{~dB}(\mathrm{~A})$.

Although performance dropped from an average of $72 \%$ correct for the 4 birds on the pretransfer session to an average of $65 \%$ correct on the transfer session, this difference was not statistically significant $[t(3)=2.99, p>.05]$. Thus there is no evidence that the loudness of the tones was controlling the discrimination.

\section{Results and Discussion}

The starlings were able to discriminate novel rising patterns from novel nonrising patterns when patterns were composed of frequencies within the frequency range of 


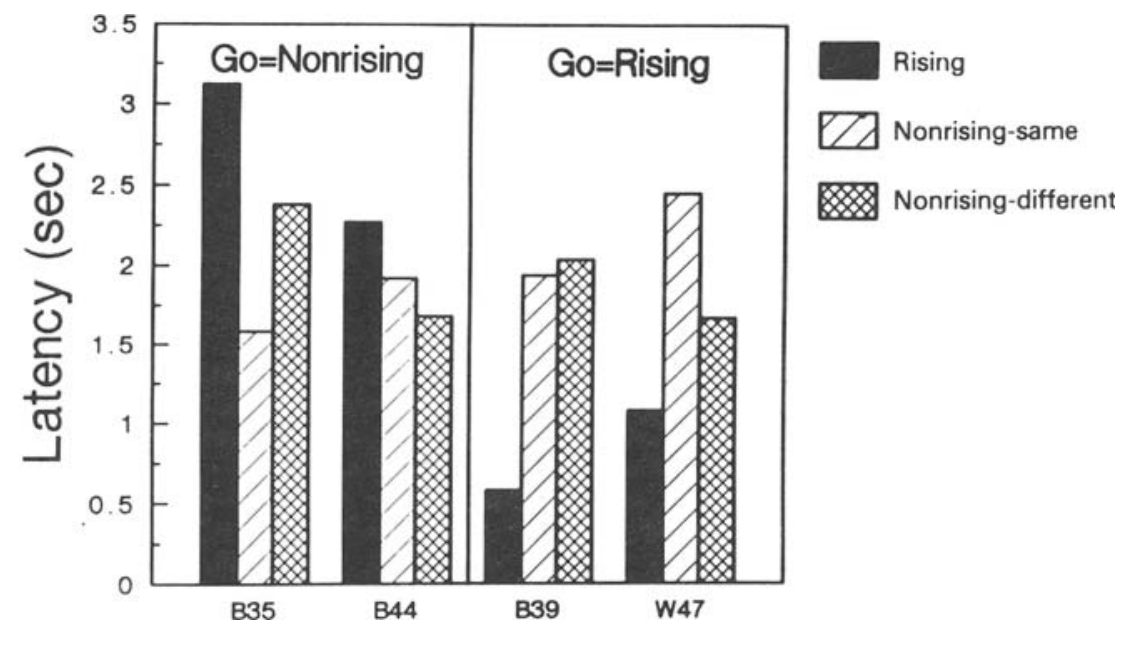

Figure 5. Mean latencies to respond to the probe patterns of Experiment 4 that were constructed of frequencies from within the baseline frequency range. Mean latencies for each type of probe pattern are shown for each bird. B35 and B44 were trained to peck for nonrising patterns; B39 and W47 were trained to peck for rising patterns.

original training. Figure 5 shows mean latency to respond to each of the three probe types for each bird. Two of the birds, B35 and B44, were trained to peck for nonrising patterns; thus nonrising latencies are shorter than rising latencies. The other birds, B 39 and $\mathrm{W} 47$, were trained to peck for rising patterns; for these birds, the latencies for rising patterns are shorter than for nonrising patterns. The starlings discriminated the patterns by pitch structure regardless of whether the nonrising patterns were exact transpositions of the baseline nonrising contours or whether they contained novel nonrising structures. Taken together, these results demonstrate that the starlings eventually learned to discriminate and categorize the patterns on the basis of their tonal structure, because the tonal structure was the only property of the patterns that remained constant in the transformed probe stimuli.

Mean latencies for the 12 probe patterns from within the frequency range of training are shown in Table 7. Generally, latencies to nonrising probes are consistently different from latencies to rising probes. Two exceptions are Probe 5 and Probe 9. For all 4 birds, the latencies for these 2 nonrising probes more closely resemble the latencies to rising probes than they do to other nonrising probes. Both of these probes began at a lower frequency $(1397 \mathrm{~Hz})$ than did any other nonrising probe within the baseline range. Thus, although the results from the other probe patterns show substantial discrimination by pitch structure, an effect of the absolute pitch of individual tones also appears. There are no consistent differences in the latencies to the 12 probe patterns from outside the frequency range of training.

Statistical analyses amply support the above results. A three-way ANOVA revealed that the interaction between contour (rising or nonrising), frequency range (inside or outside the frequency range of training), and contingency group (either rising $=$ go or rising $=$ no-go) was signifi- cant $[F(2,4)=45.52, p<.01]$. An analysis of simple effects showed that the interaction between pattern structure and frequency range was significant for both contingency groups $[F(2,4)=28.53, p<.01$, for birds in which rising $=\mathrm{go}$; and $F(2,4)=17.64, p<.05$, for birds in which rising $=$ no-go]. This indicates that the birds discriminated rising from nonrising probe patterns regardless of whether they had been trained to peck or withhold a peck for rising or nonrising patterns. The simple main effect for pattern structure was significant for both contingency groups for probe patterns composed of frequencies within the frequency range of training $[F(2,4)=$ $75.36, p<.01$, for birds in which rising $=$ go; and $F(2,4)=35.16, p<.01$, for birds in which rising $=$ no-go]. The simple main effect for pattern structure was

Table 7

Mean Latencies (in Seconds) to the 12 Probe Patterns of Experiment 1 from within the Frequency Range of Training

\begin{tabular}{|c|c|c|c|c|}
\hline \multirow[b]{2}{*}{ Probe } & \multicolumn{2}{|c|}{ No-Go $=$ Rising } & \multicolumn{2}{|c|}{ Go $=$ Rising } \\
\hline & B35 & B44 & B39 & W47 \\
\hline \multicolumn{5}{|c|}{ Rising Probes } \\
\hline 1 & 3.86 & 2.88 & 0.94 & 1.09 \\
\hline 2 & 3.60 & 3.10 & 0.44 & 0.86 \\
\hline 3 & 2.68 & 0.98 & 0.41 & 1.02 \\
\hline 4 & 2.34 & 2.11 & 0.54 & 1.37 \\
\hline \multicolumn{5}{|c|}{ Nonrising-Same Probes } \\
\hline 5 & 2.70 & 2.21 & 0.74 & 1.39 \\
\hline 6 & 0.80 & 1.98 & 2.18 & 2.75 \\
\hline 7 & 1.51 & 1.59 & 3.10 & 3.40 \\
\hline 8 & 1.36 & 1.90 & 1.73 & 2.26 \\
\hline \multicolumn{5}{|c|}{ Nonrising-Different Probes } \\
\hline 9 & 3.10 & 2.54 & 0.39 & 0.97 \\
\hline 10 & 1.86 & 1.94 & 2.92 & 1.16 \\
\hline 11 & 2.31 & 1.37 & 2.79 & 2.64 \\
\hline 12 & 2.24 & 0.88 & 2.07 & 1.92 \\
\hline
\end{tabular}


not significant for probe patterns presented outside the frequency range of training $[F(2,4)=4.31, p>.05$, and $F(2,4)=6.12, p>.05$, for birds in which rising $=$ go and rising $=$ no-go, respectively]. Simple comparisons between pattern structure types inside the frequency range of training revealed significant differences between rising pitch patterns and nonrising pitch patterns that were exact transpositions of the baseline nonrising patterns $[F(1,4)=69.52, p<.01$, and $F(1,4)=33.32, p<.01$, for birds in which rising $=$ go and rising $=$ no-go, respectively]. The differences between novel rising pitch patterns and novel nonrising pitch patterns that were not transpositions of the baseline patterns were also significant $[F(1,4)=39.20, p<.01$, and $F(1,4)=16.50, p<.05$, for birds in which rising $=$ go and rising $=$ no-go, respectively]. This indicates that the birds were able to discriminate rising from nonrising patterns within the frequency range of training, regardless of whether they were exact transpositions of baseline patterns.

The fact that the starlings were able to classify nonrising patterns with novel pitch structures shows that the birds developed a broad concept for nonrising pattern structure that was more general than the specific nonrising structures of the original baseline patterns. This observation nullifies the possibility that the birds learned the discrimination of Experiment 3 on the basis of the specific nonrising pattern structures of the baseline patterns.

The foregoing conclusions regarding pattern structure are limited to novel probe patterns that contained frequencies remaining within the original baseline frequency range. The starlings showed no ability to classify novel patterns outside the frequency range. These data provide a further example of the frequency-range constraint at work. A frequency-range constraint in songbirds has also been reported in several earlier studies (e.g., Cynx et al., 1986; Hulse \& Cynx, 1985; Page et al., 1989). Although songbirds can, in some circumstances, transpose a learned relative pitch discrimination within a familiar frequency range, the transposition is lost outside of that range.

Diminishing the predictive value of isolated pitch cues caused the birds to attend to the pitch structure that was common across the rule-based patterns. This demonstrates that, although not predisposed to do so, starlings are able to perceive the structure in sequences of pitches.

\section{GENERAL DISCUSSION}

Starlings process serial pitch patterns according to a hierarchy of perceptual strategies. First, we will describe this hierarchy; then we will turn to the evidence to support it. Finally, we relate the findings on pitch perception in starlings in operant settings to what is known about pitch perception by songbirds in more natural environments.

\section{Theory}

Perception of ordinal pitch relations among tones lies at the bottom of the hierarchy of perceptual strategies.
Here, simple relative classification occurs according to "higher than" or "lower than" rules for pairs of pitches.

The perception of pitch pattern structure lies at the top level of the hierarchy. Here, perception requires perception of ordinal relations per se, and the processing of relationships among pitch patterns composed of two or more pitches arranged in time. Within a pattern, relations among successive pattern elements may be ordinal. If so, this defines pitch pattern perception on the basis of pitch contour (the simple ups and downs of pitch). Or the serial pitch relations may be based on constant frequency ratios. In human music perception, this would define serial pitch pattern perception on the basis of melody.

There is a possibility that the hierarchy may contain a third, lowest level. Thus, starlings may first process pitch information on an absolute, nominal basis, and learn simply to "name" pitches. This would imply that the lowest level in the pitch processing hierarchy would be nonrelational; two pitches would be distinguished absolutely as if they were nominal features, not on the basis of their perceptual relationship.

It is important to point out that by "higher" and "lower" levels of the hierarchy we do not mean "more difficult" or "easier." Rather, the hierarchy is organized according to the increasing use of relational pitch information. If starlings were to demonstrate nominal perception of pitch, they would show an ability, absolute pitch, that is quite rare among humans (Ward \& Burns, 1982).

Finally, starlings apply the processes in the hierarchy from the bottom up. They first isolate and learn ordinal relations among pairs of pitches. The subsequent perception (and differentiation) of pitch pattern structure requires both the perception of ordinal pitch relations and the perception of temporal order among successive pitches within patterns. Again, the possibility remains that pitch processing may begin, in fact, with simple classification of pitches on an absolute basis and proceed from there to the two higher stages on the basis of relational learning.

We make no claim about the true nature of the processes underlying the hierarchy. It is possible that the hierarchy is, in fact, based primarily on associative or perceptual processing of auditory stimulus patterns at some high central level. It is also possible, as Marler (1982) and Page et al. (1989) have pointed out, that the hierarchy may represent different levels of attentional preference for pitch features.

\section{Evidence}

What is the evidence for the assertion that starlings use a hierarchy for serial pitch processing? Perception of high-low ordinal relations certainly was used to discriminate the patterns in Experiments 1 and 2. In Experiment 1, the birds transferred most easily to problems that retained identical high-low pitch relationships among their initial tones. In Experiment 2, the starlings transferred well to novel patterns that retained the high-low pitch relationships between initial tones, and failed to transfer when 
that relationship was reversed. These data show a strong propensity for the starlings to perceive pitch relationally.

When the experimental conditions ruled out the use of simple ordinal relations as a discriminative cue, the starlings showed that they could perceive structure in pitch patterns and classify those patterns according to generalizable rules that define the patterns. That is, they showed that they could process pitch relations at the highest level of the hierarchy. The starlings did so in Experiment 3, in which a new stimulus context obscured the adequacy of individual pattern tones as a discriminative cue. Four of the initial six frequencies in Experiment 3-1480, 1661,1865 , and $2093 \mathrm{~Hz}$-initiated both rising and nonrising patterns (cf. Figure 1). For only 2 of the 12 patterns-the rising patterns beginning on 1175 and $1319 \mathrm{~Hz}$-was the initial tone perfectly correlated with pitch structure. This stimulus context produced substantial difficulty, but the birds eventually learned to classify rising and nonrising patterns.

Of course, it is entirely possible that the birds simply sharpened their use of individual pitch cues over the course of Experiment 3 and learned to discriminate among the 12 patterns on that basis. However, further convincing evidence that the starlings were learning to classify the patterns in Experiment 3 on the basis of formal pattern structure, as distinguished from individual tones, comes from Experiment 4 . Here, the starlings properly classified unreinforced rising and nonrising exemplars that they had never heard before. Furthermore, they did so regardless of whether the pitch structures of nonrising patterns were familiar or novel-indicating that they had developed a generalizable concept of "nonrising."

Although there is abundant evidence for processing based on pitch relations at the higher levels of the hierarchy, the role of absolute pitch perception (at the bottom of the hierarchy) is less clear. The frequency-range constraint is a case of absolute pitch perception for artificial stimuli. Starlings (and mockingbirds and cowbirds) encode information about the absolute frequency range of the tones comprising pitch patterns, and this absolute pitch information constrains generalization to novel patterns outside the initial range. As in earlier work (Cynx et al., 1986; Hulse et al., 1984; Page et al., 1989), Experiment 4 revealed a failure to generalize pitch pattern discrimination when rising and nonrising probe pattern frequencies were shifted outside the frequency range in which the original pattern discrimination had taken place. Despite this evidence, we do not know for sure if absolute pitch perception was a necessary initial requisite for successful discrimination in the experiments reported here.

Taken together, these and earlier results suggest that individual pattern elements play a crucial role in the development of discrimination of pitch patterns by songbirds. If songbirds are asked to learn to discriminate among pitch patterns solely on the basis of pattern structure-that is, if they are asked to process pitch patterns initially at the topmost level of the hierarchy-they fail to do so despite many thousands of trials (Page et al.,
1989). Instead, if pattern element and pattern structure cues are both available, there is a strong predilection for the use of pattern element cues-that is, to process at a lower level in the hierarchy. That was true in the present experiment, and it has also been true in earlier work involving several species of songbirds (Cynx et al., 1986; Hulse, 1989; Hulse \& Cynx, 1985, 1986; Hulse et al., 1984; Hulse et al., 1990), parakeets (Dooling, Brown, Park, Okanoya, \& Soli, 1987; Park \& Dooling, 1985), rats (D'Amato \& Salmon, 1984), and cebus monkeys (D'Amato \& Colombo, 1988; D'Amato \& Salmon, 1984). Only when the experimental conditions severely constrain the use of pattern element cues do starlings display (or develop) the capacity for perception of pattern structure at higher levels. This is in direct contrast to the perception of pitch patterns by adult humans, in which the perception of structure dominates the perception of elements (Espinoza-Varas \& Watson, 1989). Of course, these conclusions regarding comparisons between humans and nonhumans must be hedged until species are tested on tasks that are directly comparable. It is also possible that comparisons between infant humans and nonhumans may be especially appropriate and instructive.

\section{Perception of Bird Song}

Studies of the perception of bird song converge with the operant studies to show that songbirds make use of both absolute pitch perception and perception of pitch relations among song elements. Chickadees showed a decrease in responding to fee-bee songs that were shifted in either absolute or relative pitch (Weisman \& Ratcliffe, 1989). Interestingly, the birds showed changes in responses to the absolute pitch shift that were different from those to the relative pitch shift, indicating that these two parameters may code different content for this species.

Playback studies have revealed some results that parallel the phenomenon of the frequency-range constraint. Whitethroated sparrows (Brooks \& Falls, 1975) have shown a change in responding to neighbors' songs when those songs were shifted in pitch by $15 \%$. A $15 \%$ shift did affect neighbor recognition. Nelson (1988) has found that a $15 \%$ shift in pitch did not alter the response of field sparrows to conspecific song. An $18 \%$ shift did alter the response, however. Thus, songbirds do show a tolerance for variance in the absolute pitch of song. This parallels the results of transfers to within-range probes. As in the frequency-range constraint, song shifted above a certain threshold elicits an altered response. In the case of bird song, the frequency-range constraint is probably the result of sensitivity to the range of natural variation of song. Pitch as a parameter of bird song may play a dual role. It may serve as a marker of individuals as well as species (Brooks \& Falls, 1975). That is, there may be a frequency range that is characteristic of a species. An absolute frequency within that range would identify an individual. This would be demonstrated by a greater tolerance to shifts in pitch in discriminating conspecifics as opposed to individuals. This idea remains to be tested. 


\section{REFERENCES}

Adret-Hausberger, M. (1988). Song differentiation and population structure: The example of the whistled songs in an introduced population of European starlings Sturnus vulgaris in Australia. Ethology, 79, 104-115.

Attneave, F., \& Olson, R. K. (1971). Pitch as a medium: A new approach to psychophysical scaling. American Journal of Psychology, 84, 147-166.

Bregman, A. S., \& Campbell, J. (1971). Primary auditory stream segregation and perception of order in rapid sequences of tones. Journal of Experimental Psychology, 89, 244-249.

Brooks, R. J., \& FALLS, J. B. (1975). Individual recognition by song in white-throated sparrows: III. Song features used in individual recognition. Canadian Journal of Zoology, 53, 1749-1761.

Cerella, J. (1979). Visual classes and natural categories in the pigeon. Joumal of Experimental Psychology: Human Perception \& Performance, 5, 68-77.

CynX, J., Hulse, S. H., \& Polyzors, S. (1986). A psychophysical measure of pitch discrimination loss resulting from a frequency range constraint in European starlings (Sturnus vulgaris). Journal of Experimental Psychology: Animal Behavior Processes, 12, 394-402.

D'Amato, M. R., \& Colomвo, M. (1988). On tonal pattern perception in monkeys (Cebus apella). Animal Learning \& Behavior, 16, 417-424.

D'Amato, M. R., \& Salmon, D. P. (1982). Tune discrimination in monkeys (Cebus apella) and in rats. Animal Learning \& Behavior, 10, 126-134.

D'Amato, M. R., \& Salmon, D. P. (1984). Processing of complex auditory stimuli (tunes) by rats and monkeys (Cebus apella). Animal Learning \& Behavior, 12, 184-194.

DEUTsCh, D. (Ed.) (1982). The psychology of music. New York: Academic Press.

DivenYI, P. L., \& HiRSH, I. J. (1978). Some figural properties of auditory patterns. Journal of the Acoustical Society of America, 64, 1369-1385.

Dooling, R. J., Brown, S. D., Park, T. J., Okanoya, K., \& Soli, S. (1987). Perceptual organization of acoustic stimuli by budgerigars (Melopsittacus undulatus): I. Pure tones. Joumal of Comparative Psychology, 101, 139-149.

Dooung, R. J., Okanoya, K., Downing, J., \&ulse, S. [H.] (1986). Hearing in the starling (Stumus vulgaris): Absolute thresholds and critical ratios. Bulletin of the Psychonomic Society, 24, 462-464.

Dowling, W. J., \& HARWOOD, D. L. (1986). Music cognition. New York: Academic Press.

Espinoza-VAras, B., \& Watson, C. S. (1986). Temporal discrimination for single components of nonspeech auditory patterns. Joumal of the Acoustical Society of America, 80, 1685-1694.

Espinoza-Varas, B., \& Watson, C. S. (1989). Perception of complex auditory patterns by humans. In R. J. Dooling \& S. H. Hulse (Eds.), The comparative psychology of audition: Perceiving complex sounds (pp. 67-94). Hillsdale, NJ: Erlbaum.

GARNER, W. R. (1974). The processing of information and structure. Potomac, MD: Erlbaum.

GARNER, W. R., \& GoTTWALD, R. L. (1968). The perception and learning of temporal patterns. Quarterly Journal of Experimental Psychology, 20, 97-109.

GREEN, D. M. (1976). An introduction to hearing. Hillsdale, NJ: Erlbaum.

Harlow, H. F. (1949). The formation of learning sets. Psychological Review, 56, 51-65.

Herman, L. H., \& Arbeit, W. R. (1973). Stimulus control and auditory discrimination learning sets in the bottlenose dolphin. Journal of the Experimental Analysis of Behavior, 19, 379-394.

HeRrastein, R. J., \& Loveland, D. H. (1964). Complex visual concept in the pigeon. Science, 146, 549-551.
Herrnstein, R. J., Loveland, D. H., \& Cable, C. (1976). Natural concepts in the pigeon. Journal of Experimental Psychology: Animal Behavior Processes, 2, 285-311.

HULSE, S. H. (1989). Comparative psychology and pitch pattern perception in songbirds. In R. J. Dooling \& S. H. Hulse (Eds.), The comparative psychology of audition: Perceiving complex sounds (pp. 331349). Hillsdale, NJ: Erlbaum.

HULSE, S. H., \& CYNX, J. (1985). Relative pitch perception is constrained by absolute pitch in songbirds (Mimus, Molothrus, and Sturnus). Journal of Comparative Psychology, 99, 176-196.

Hulse, S. H., CYNX, J. (1986). Interval and contour in serial pitch perception by a passerine bird, the European starling (Sturnus vulgaris). Journal of Comparative Psychology, 100, 215-228.

Hulse, S. H., CynX, J., \& Humpal, J. (1984). Absolute and serial pitch discrimination in serial perception by birds. Journal of Experimental Psychology: General, 113, 38-54.

Hulse, S. H., \& PAGE, S. C. (1988). Toward a comparative psychology of music perception. Music Perception, 5, 427-452.

Hulse, S. H., Page, S. H., \& Braten, R. F. (1990). An integrative approach to auditory perception by songbirds. In M. Berkley \& W. C. Stebbins (Eds.), Comparative perception (Vol. 2, pp. 3-34). New York: Wiley.

MARLer, P. (1982). Avian and primate communication: The problem of natural categories. Neuroscience \& Biobehavioral Reviews, 6, 87-94.

NELSON, D. A. (1988). Feature weighting in species song recognition by the field sparrow (Spizella pusilla). Behaviour, 106, 158-182.

PAGE, S. C., Hulse, S. H., \& CYNX, J. (1989). Relative pitch perception in the European starling (Sturnus vulgaris): Further evidence for an elusive phenomenon. Journal of Experimental Psychology: Animal Behavior Processes, 15, 137-146.

Park, T. J., \& Dooling, R. J. (1985). Perception of species-specific contact calls by budgerigars (Melopsittacus undulatus). Journal of Comparative Psychology, 99, 391-402.

ROYER, F. L., \& GARNER, W. R. (1966). Response uncertainty and perceptual difficulty of auditory temporal patterns. Perception \& Psychophysics, 1, 41-47.

Royer, F. L., Garner, W. R. (1970). Perceptual organization of nine-element auditory temporal patterns. Perception \& Psychophysics, 7, 115-120.

SLOTNICK, B. M., \& KATZ, H. M. (1974). Olfactory learning-set formation in rats. Science, $185,796-798$.

TREHUB, S. E. (1987). Infants' perception of musical patterns. Perception \& Psychophysics, 41, 635-641.

WARD, W. D., BURNS, E. M. (1982). Absolute pitch. In D. Deutsch (Ed.), The psychology of music. New York: Academic Press.

Watson, C. S., Kelly, W. J., \& Woton, H. W. (1976). Factors in the discrimination of tonal patterns: II. Selective attention and learning under various conditions of stimulus uncertainty. Journal of the Acoustical Society of America, 60, 1176-1186.

Watson, C. S., Wroton, H. W., Kelly, W. J., \& Benbasset, C. A. (1975). Factors in the discrimination of tonal patterns: I. Component frequency, temporal position, and silent intervals. Journal of the Acoustical Society of America, 57, 1175-1185.

Weisman, R., RATCLIFF, L. (1989). Absolute and relative pitch processing in black-capped chickadees, Parus atricapillus. Animal Behaviour, 38, 685-692.

\section{NOTE}

1. Many acoustic events can produce the psychological experience of pitch, and there are many theoretical accounts for it (Green, 1976). For purposes of this paper, we choose the operational definition that the pitch of a tone is defined by the frequency of a matching sine tone.

(Manuscript received January 2, 1990; revision accepted for publication May 30, 1990.) 